\title{
ON THE RESTRICTED PARTITION FUNCTION VIA DETERMINANTS WITH BERNOULLI POLYNOMIALS. II
}

\author{
MIRCEA CIMPOEAŞ
}

\begin{abstract}
Let $r \geq 1$ be an integer, $\mathbf{a}=\left(a_{1}, \ldots, a_{r}\right)$ a vector of positive integers, and let $D \geq 1$ be a common multiple of $a_{1}, \ldots, a_{r}$. We prove that if $D=1$ or $D$ is a prime number then the restricted partition function $p_{\mathbf{a}}(n):=$ the number of integer solutions $\left(x_{1}, \ldots, x_{r}\right)$ to $\sum_{j=1}^{r} a_{j} x_{j}=n$, with $x_{1} \geq 0, \ldots, x_{r} \geq 0$, can be computed by solving a system of linear equations with coefficients that are values of Bernoulli polynomials and Bernoulli-Barnes numbers.
\end{abstract}

\section{INTRODUCTION}

Let $\mathbf{a}:=\left(a_{1}, a_{2}, \ldots, a_{r}\right)$ be a sequence of positive integers, $r \geq 1$. The restricted partition function associated to $\mathbf{a}$ is $p_{\mathbf{a}}: \mathbb{N} \rightarrow \mathbb{N}, p_{\mathbf{a}}(n):=$ the number of integer solutions $\left(x_{1}, \ldots, x_{r}\right)$ of $\sum_{i=1}^{r} a_{i} x_{i}=n$ with $x_{i} \geq 0$. Let $D$ be a common multiple of $a_{1}, \ldots, a_{r}$. According to [5], $p_{\mathbf{a}}(n)$ is a quasi-polynomial of degree $r-1$, with the period $D$, i.e.

$$
p_{\mathbf{a}}(n)=d_{\mathbf{a}, r-1}(n) n^{r-1}+\cdots+d_{\mathbf{a}, 1}(n) n+d_{\mathbf{a}, 0}(n), \quad \text { for all } n \geq 0,
$$

where $d_{\mathbf{a}, m}(n+D)=d_{\mathbf{a}, m}(n)$, for all $0 \leq m \leq r-1, n \geq 0$, and $d_{\mathbf{a}, r-1}(n)$ is not identically zero. The restricted partition function $p_{\mathbf{a}}(n)$ was studied extensively in the literature, starting with the works of Sylvester [15] and Bell [5]. Popoviciu [1] gave a precise formula for $r=2$. Recently, Bayad and Beck [4, Theorem 3.1] proved an explicit expression of $p_{\mathbf{a}}(n)$ in terms of Bernoulli-Barnes polynomials and the Fourier-Dedekind sums, in the case that $a_{1}, \ldots, a_{r}$ are pairwise coprime. In [6], we proved that the computation of $p_{\mathbf{a}}(n)$ can be reduced to solving the linear congruency $a_{1} j_{1}+\cdots+a_{r} j_{r} \equiv n(\bmod D)$ in the range $0 \leq j_{1} \leq \frac{D}{a_{1}}, \ldots, 0 \leq$ $j_{r} \leq \frac{D}{a_{r}}$. In [8], we proved that if a determinant $\Delta_{r, D}$, which depends only on $r$ and $D$, with entries consisting in values of Bernoulli polynomials is nonzero, then $p_{\mathbf{a}}(n)$ can be computed in terms of values of Bernoulli polynomials and BernoulliBarnes numbers. The aim of this paper is to tackle the same problem, from another perspective that relays on the arithmetic properties of Bernoulli polynomials.

2010 Mathematics Subject Classification. Primary 11P81; Secondary 11B68, 11P82.

Key words and phrases. Restricted partition function; Bernoulli polynomial; Bernoulli-Barnes numbers. 
First we recall some definitions. The Barnes zeta function associated to a and $w>0$ is

$$
\zeta_{\mathbf{a}}(s, w):=\sum_{n=0}^{\infty} \frac{p_{\mathbf{a}}(n)}{(n+w)^{s}}, \quad \operatorname{Re} s>r ;
$$

see [3] and [13] for further details. It is well known that $\zeta_{\mathbf{a}}(s, w)$ is meromorphic on $\mathbb{C}$ with poles at most in the set $\{1, \ldots, r\}$. We consider the function

$$
\zeta_{\mathbf{a}}(s):=\lim _{w \searrow 0}\left(\zeta_{\mathbf{a}}(s, w)-w^{-s}\right) .
$$

In [6. Lemma 2.6], we proved that

$$
\zeta_{\mathbf{a}}(s)=\frac{1}{D^{s}} \sum_{m=0}^{r-1} \sum_{v=1}^{D} d_{\mathbf{a}, m}(v) D^{m} \zeta\left(s-m, \frac{v}{D}\right),
$$

where

$$
\zeta(s, w):=\sum_{n=0}^{\infty} \frac{1}{(n+w)^{s}}, \quad \operatorname{Re} s>1,
$$

is the Hurwitz zeta function; see also [7]. The Bernoulli numbers $B_{j}$ are defined by

$$
\frac{z}{e^{z}-1}=\sum_{j=0}^{\infty} B_{j} \frac{z^{j}}{j !},
$$

$B_{0}=1, B_{1}=-\frac{1}{2}, B_{2}=\frac{1}{6}, B_{4}=-\frac{1}{30}$, and $B_{n}=0$ if $n$ is odd and greater than 1 . The Bernoulli polynomials are defined by

$$
\frac{z e^{x z}}{\left(e^{z}-1\right)}=\sum_{n=0}^{\infty} B_{n}(x) \frac{z^{n}}{n !} .
$$

They are related to the Bernoulli numbers by

$$
B_{n}(x)=\sum_{k=0}^{n}\left(\begin{array}{l}
n \\
k
\end{array}\right) B_{n-k} x^{k} .
$$

It is well known (see for instance [2, Theorem 12.13]) that

$$
\zeta(-n, w)=-\frac{B_{n+1}(w)}{n+1}, \quad \text { for all } n \in \mathbb{N}, w>0 .
$$

The Bernoulli-Barnes polynomials are defined by

$$
\frac{z^{r} e^{x z}}{\left(e^{a_{1} z}-1\right) \cdots\left(e^{a_{r} z}-1\right)}=\sum_{j=0}^{\infty} B_{j}(x ; \mathbf{a}) \frac{z^{j}}{j !} .
$$

The Bernoulli-Barnes numbers are defined by

$$
B_{j}(\mathbf{a}):=B_{j}(0 ; \mathbf{a})=\sum_{i_{1}+\cdots+i_{r}=j}\left(\begin{array}{c}
j \\
i_{1}, \ldots, i_{r}
\end{array}\right) B_{i_{1}} \cdots B_{i_{r}} a_{1}^{i_{1}-1} \cdots a_{r}^{i_{r}-1} .
$$

According to [12, Formula (3.10)], the formula

$$
\zeta_{\mathbf{a}}(-n, w)=\frac{(-1)^{r} n !}{(n+r) !} B_{r+n}(w ; \mathbf{a})
$$


holds for all $n \in \mathbb{N}$. From 1.2 and $(1.6)$, it follows that

$$
\zeta_{\mathbf{a}}(-n)=\frac{(-1)^{r} n !}{(n+r) !} B_{r+n}(\mathbf{a}), \quad \text { for all } n \geq 1 .
$$

From (1.3), 1.5), and (1.7), it follows that

$$
\sum_{m=0}^{r-1} \sum_{v=1}^{D} d_{\mathbf{a}, m}(v) D^{n+m} \frac{B_{n+m+1}\left(\frac{v}{D}\right)}{n+m+1}=\frac{(-1)^{r-1} n !}{(n+r) !} B_{r+n}(\mathbf{a}), \quad \text { for all } n \geq 1 .
$$

Let $\underline{\alpha}: \alpha_{1}<\alpha_{2}<\cdots<\alpha_{r D}$ be a sequence of integers with $\alpha_{1} \geq 2$. Substituting $n$ with $\alpha_{j}-1,1 \leq j \leq r D$, in 1.8 and multiplying by $D$, we obtain the system of linear equations

$$
\sum_{m=0}^{r-1} \sum_{v=1}^{D} d_{\mathbf{a}, m}(v) \frac{D^{\alpha_{j}+m} B_{\alpha_{j}+m}\left(\frac{v}{D}\right)}{\alpha_{j}+m}=\frac{(-1)^{r-1}\left(\alpha_{j}-1\right) ! D}{\left(\alpha_{j}+r-1\right) !} B_{\alpha_{j}+r-1}(\mathbf{a}), \quad 1 \leq j \leq r D,
$$

which has the determinant

$$
\begin{gathered}
\Delta_{r, D}(\underline{\alpha}):= \\
\left|\begin{array}{ccccccc}
\frac{D^{\alpha_{1}} B_{\alpha_{1}}\left(\frac{1}{D}\right)}{\alpha_{1}} & \ldots & \frac{D^{\alpha_{1}} B_{\alpha_{1}}(1)}{\alpha_{1}} & \ldots & \frac{D^{\alpha_{1}} B_{\alpha_{1}+r-1}\left(\frac{1}{D}\right)}{\alpha_{1}+r-1} & \ldots & \frac{D^{\alpha_{1}} B_{\alpha_{1}+r-1}(1)}{\alpha_{1}+r-1} \\
\frac{D^{\alpha_{2}} B_{\alpha_{2}}\left(\frac{1}{D}\right)}{\alpha_{2}} & \ldots & \frac{D^{\alpha_{2}} B_{\alpha_{2}}(1)}{\alpha_{2}} & \ldots & \frac{D^{\alpha_{2}} B_{\alpha_{2}+r-1}\left(\frac{1}{D}\right)}{\alpha_{2}+r-1} & \ldots & \frac{D^{\alpha_{2}} B_{\alpha_{2}+r-1}(1)}{\alpha_{2}+r-1} \\
\vdots & \ddots & \vdots & \ddots & \vdots & \ddots & \vdots \\
\frac{D^{\alpha_{r D}} B_{\alpha_{r D}\left(\frac{1}{D}\right)}}{\alpha_{r D}} & \ldots & \frac{D^{\alpha_{r D}} B_{\alpha_{r D}}(1)}{\alpha_{r D}} & \ldots & \frac{D^{\alpha_{r D} B_{\alpha_{r D}+r-1}\left(\frac{1}{D}\right)}}{\alpha_{r D}+r-1} & \ldots & \frac{D^{\alpha_{r D} B_{\alpha_{r D}+r-1}(1)}}{\alpha_{r D}+r-1}
\end{array}\right| .
\end{gathered}
$$

Note that, with the notation given in [8, (2.10)], we have

$$
\Delta_{r, D}=\Delta_{r, D}(0,1, \ldots, r D-1) \text {. }
$$

Here we omit the condition $\alpha_{1} \geq 2$.

Proposition 1.1. With the above notation, if $\Delta_{r, D}(\underline{\alpha}) \neq 0$ then

$$
d_{\mathbf{a}, m}(v)=\frac{\Delta_{r, D}^{m, v}(\underline{\alpha})}{\Delta_{r, D}(\underline{\alpha})}, \quad \text { for all } 1 \leq v \leq D, 0 \leq m \leq r-1,
$$

where $\Delta_{r, D}^{m, v}(\underline{\alpha})$ is the determinant obtained from $\Delta_{r, D}(\underline{\alpha})$, as defined in $(1.9)$, by replacing the $(m D+v)$-th column with the column

$$
\left(\frac{(-1)^{r-1}\left(\alpha_{j}-1\right) ! D}{\left(\alpha_{j}+r-1\right) !} B_{\alpha_{j}+r-1}(\mathbf{a})\right)_{1 \leq j \leq r D-1} .
$$

Moreover,

$$
p_{\mathbf{a}}(n)=\frac{1}{\Delta_{r, D}(\underline{\alpha})} \sum_{m=0}^{r-1} \Delta_{r, D}^{m, v}(\underline{\alpha}) n^{m}, \quad \text { for all } n \in \mathbb{N} .
$$

Proof. It follows from 1.8 and 1.9 by Cramer's rule. The last assertion follows from 1.1 .

Our main theorem is the following. 
Theorem 1.2. Let $r \geq 1$ and let $D=1$ or $D \geq 2$ be a prime number. There exists a sequence of integers $\underline{\alpha}: \alpha_{1}<\alpha_{2}<\cdots<\alpha_{r D}, \alpha_{1} \geq 2$, such that $\Delta_{r, D}(\underline{\alpha}) \neq 0$. In particular, we can compute $p_{\mathbf{a}}(n)$ in terms of values of Bernoulli polynomials and Bernoulli-Barnes numbers.

We believe that the result holds for any integer $D \geq 1$. Unfortunately, our method based on $p$-adic valuations and congruences for Bernoulli numbers and for the values of Bernoulli polynomials, is not refined enough to prove it.

\section{Properties of Bernoulli polynomials}

We recall several properties of the Bernoulli polynomials. We have that

$$
B_{n}(1-x)=(-1)^{n} B_{n}(x), \quad \text { for all } n \in \mathbb{N} .
$$

For any integers $n \geq 1$ and $1 \leq v \leq D$, using (1.4), we let

$$
\tilde{B}_{n}(x):=D^{n}\left(B_{n}(x)-B_{n}\right)=\sum_{j=1}^{n-1}\left(\begin{array}{l}
n \\
j
\end{array}\right) D^{j}(x D)^{n-j} .
$$

According to [1, Theorem 1], we have that

$$
\tilde{B}_{n}\left(\frac{v}{D}\right) \in \mathbb{Z}, \quad \text { for all } 1 \leq v \leq D .
$$

According to a result of T. Clausen and C. von Staudt (see [9, 14]), we have that

$$
B_{2 n}=A_{2 n}-\sum_{p-1 \mid 2 n} \frac{1}{p}, \quad \text { for all } n \geq 1,
$$

where $A_{2 n} \in \mathbb{Z}$ and the sum is over all the primes $p$ such that $p-1 \mid 2 n$.

Let $p$ be a prime. For any integer $a$, the $p$-adic order of $a$ is $v_{p}(a):=\max \{k$ : $\left.p^{k} \mid a\right\}$, if $a \neq 0$, and $v_{p}(0)=\infty$. For $q=\frac{a}{b} \in \mathbb{Q}$, the $p$-adic order of $q$ is $v_{p}(q):=v_{p}(a)-v_{p}(b)$. Note that 2.4 implies

$$
v_{p}\left(B_{2 n}\right)= \begin{cases}-1, & p-1 \mid 2 n ; \\ \geq 0, & p-1 \nmid 2 n .\end{cases}
$$

Lemma 2.1. For any integer $n \geq 1$, we have that:

(1) $\tilde{B}_{n}\left(\frac{1}{2}\right)=0$ if $n$ is odd, and $\tilde{B}_{n}\left(\frac{1}{2}\right) \equiv 1(\bmod 2)$ if $n$ is even.

(2) If $p$ is a prime, then $\tilde{B}_{n}\left(\frac{v}{p}\right) \equiv v^{n}(\bmod p)$, for all $1 \leq v \leq p-1$.

Proof. (1) From (2.1) it follows that $B_{n}\left(\frac{1}{2}\right)=0$ if $n$ is odd. Hence, as $B_{n}=0$, we get

$$
\tilde{B}_{n}\left(\frac{1}{2}\right)=D^{n}\left(B_{n}\left(\frac{1}{2}\right)-B_{n}\right)=0 .
$$

Assume that $n$ is even. According to 2.2 , we have

$$
\tilde{B}_{n}\left(\frac{1}{2}\right)=\sum_{j=0}^{n}\left(\begin{array}{l}
n \\
j
\end{array}\right) B_{j} 2^{j}
$$


Since $2 \mid 2 n B_{1}=-n$ and $v_{2}\left(2^{j} B_{j}\right) \geq 1$ for any $j \geq 2$, the conclusion follows immediately.

(2) According to 2.2), we have that

$$
\tilde{B}_{n}\left(\frac{a}{p}\right)=\sum_{j=0}^{n}\left(\begin{array}{l}
n \\
j
\end{array}\right) B_{j} v^{n-j} p^{j}
$$

From 2.5, we have that $v_{p}\left(p^{j} B_{j}\right) \geq 1$ for $j \geq 1$, hence the conclusion follows immediately.

Lemma 2.2. If $p$ is a prime such that $p \nmid D$ then

$$
\tilde{B}_{p}\left(\frac{v}{D}\right) \equiv 0 \quad(\bmod p), \quad \text { for all } 1 \leq v \leq D-1 .
$$

Proof. We have that

$$
\tilde{B}_{p}\left(\frac{v}{D}\right)=\sum_{j=0}^{p}\left(\begin{array}{l}
p \\
j
\end{array}\right) B_{j} v^{p-j} D^{j}
$$

Since $v_{p}\left(B_{j}\right) \geq 0$ for $j \leq p-2$, it follows that

$$
v_{p}\left(\left(\begin{array}{c}
p \\
j
\end{array}\right) B_{j}\right) \geq 1, \quad \text { for all } 1 \leq j \leq p-2 .
$$

On the other hand, it follows from (2.4) that

$$
v^{p}+\left(\begin{array}{c}
p \\
p-1
\end{array}\right) B_{p-1} v D^{p-1} \equiv v^{p}-v D^{p-1} \equiv v^{p}-v \equiv 0 \quad(\bmod p) .
$$

Hence, we get the required result.

\section{Preliminary Results}

Proposition 3.1 (Case $D=1$ ). Let $p_{1}<p_{2}<\cdots<p_{r}$ be some primes such that $p_{1}>2$ and $p_{j+1}-p_{j}>r$, for all $1 \leq j \leq r-1$. Let $\alpha_{j}:=p_{j}-j, 1 \leq j \leq r$. We have that $\Delta_{r, 1}(\underline{\alpha}) \neq 0$.

Proof. Note that 2.1) implies $B_{n}(1)=B_{n}$ for any $n \geq 2$. It follows that

$$
\Delta_{r, 1}(\underline{\alpha})=\left|\begin{array}{cccc}
\frac{B_{\alpha_{1}}}{\alpha_{1}} & \frac{B_{\alpha_{1}+1}}{\alpha_{1}+1} & \ldots & \frac{B_{\alpha_{1}+r-1}}{\beta_{1}+r-1} \\
\frac{B_{\alpha_{2}}}{\alpha_{2}} & \frac{B_{\alpha_{1}+1}}{\alpha_{2}+1} & \ldots & \frac{B_{\alpha_{2}+r-1}}{\alpha_{2}+r-1} \\
\vdots & \vdots & \ddots & \vdots \\
\frac{B_{\alpha_{r}}}{\alpha_{r}} & \frac{B_{\alpha_{1}+1}}{\alpha_{r}+1} & \ldots & \frac{B_{\alpha_{r}+r-1}}{\alpha_{r}+r-1}
\end{array}\right| .
$$

From (2.4) it follows that $v_{p_{j}}\left(B_{\alpha_{j}+j-1}\right)=-1$ and $v_{p_{j}}\left(B_{\alpha_{j}+k-1}\right) \geq 0$, for all $1 \leq k \leq$ $r$ with $k \neq j$. Moreover, if $1 \leq \ell<j \leq r$, then, by hypothesis, $v_{p_{j}}\left(B_{\alpha_{\ell}+k-1}\right) \geq 0$ for any $1 \leq k \leq r$ (we implicitly used the fact that $B_{n}=0$ if $n \geq 3$ is odd). It follows that, in the expansion of $\Delta_{r, 1}(\underline{\alpha})$ written in $(3.1)$, the term

$$
\prod_{j=1}^{r} \frac{D^{\alpha_{j}+j-1} B_{\alpha_{j}+j-1}}{\alpha_{j}+j-1}
$$

cannot be simplified, hence $\Delta_{r, 1}(\underline{\alpha}) \neq 0$. 
In the following, we assume that $D \geq 2$ and we consider the determinant $\tilde{\Delta}_{r, D}(\underline{\alpha}):=$

$$
\left|\begin{array}{ccccccc}
\frac{\tilde{B}_{\alpha_{1}}\left(\frac{1}{D}\right)}{\alpha_{1}} & \ldots & \frac{\tilde{B}_{\alpha_{1}}\left(\frac{D-1}{D}\right)}{\alpha_{1}} & \ldots & \frac{\tilde{B}_{\alpha_{1}+r-1}\left(\frac{1}{D}\right)}{\alpha_{1}+r-1} & \ldots & \frac{\tilde{B}_{\alpha_{1}+r-1}\left(\frac{D-1}{D}\right)}{\alpha_{1}+r-1} \\
\frac{\tilde{B}_{\alpha_{2}}\left(\frac{1}{D}\right)}{\alpha_{2}} & \ldots & \frac{\tilde{B}_{\alpha_{2}}\left(\frac{b-1}{D}\right)}{\alpha_{2}} & \ldots & \frac{\tilde{B}_{\alpha_{2}+r-1}\left(\frac{1}{D}\right)}{\alpha_{2}+r-1} & \ldots & \frac{\tilde{B}_{\alpha_{2}+r-1}\left(\frac{D-1}{D}\right)}{\alpha_{2}+r-1} \\
\vdots & \ddots & \vdots & \ddots & \vdots & \ddots & \vdots \\
\frac{\tilde{B}_{\alpha_{r D-r}\left(\frac{1}{D}\right)}}{\alpha_{r D-r}} & \ldots & \frac{\tilde{B}_{\alpha_{r D-r}\left(\frac{D-1}{D}\right)}}{\alpha_{r D-r}} & \ldots & \frac{\tilde{B}_{\alpha_{r D-r}+r-1}\left(\frac{1}{D}\right)}{\alpha_{r D-r}+r-1} & \ldots & \frac{\tilde{B}_{\alpha_{r D-r}+r-1}\left(\frac{D-1}{D}\right)}{\alpha_{r D-r}+r-1}
\end{array}\right| .
$$

Let $p_{1}<p_{2}<\cdots<p_{r}$ be some primes such that

$$
p_{1} \geq \alpha_{r(D-1)}+r \quad \text { and } \quad p_{j+1}-p_{j}>r, \quad \text { for all } 1 \leq j \leq r-1 .
$$

We let

$$
\alpha_{r D-r+j}:=p_{j}-j, \quad \text { for all } 1 \leq j \leq r .
$$

According to Lemma 2.2 and $(3.3)$, we have that

$$
v_{p_{\ell}}\left(\frac{\tilde{B}_{\alpha_{r D-r+j}+j}\left(\frac{v}{D}\right)}{\alpha_{r D-r+j}+j}\right) \geq 0, \quad \text { for all } 1 \leq j, \ell \leq r, 1 \leq v \leq D-1 .
$$

On the other hand, since $p_{j} \geq \alpha_{r(D-1)}+r$, from Lemma 2.2 it follows that

$$
v_{p_{\ell}}\left(\frac{D^{\alpha_{t}+j} B_{\alpha_{t}+j}}{\alpha_{t}+j}\right) \geq 0, \quad v_{p_{\ell}}\left(\frac{\tilde{B}_{\alpha_{t}+j}\left(\frac{v}{D}\right)}{\alpha_{t}+j}\right) \geq 0,
$$

for all $1 \leq j, \ell \leq r, 1 \leq t \leq r(D-1), 1 \leq v \leq D-1$. Also, from 2.5 and (3.3), it follows that

$$
v_{p_{\ell}}\left(\frac{B_{\alpha_{r D-r+j}+j}\left(\frac{v}{D}\right)}{\alpha_{r D-r+j}+j}\right) \geq 0, \quad v_{p_{j}}\left(\frac{B_{\alpha_{r D-r+j}+j}\left(\frac{v}{D}\right)}{\alpha_{r D-r+j}+j}\right)=-1,
$$

for $1 \leq j, \ell \leq r, j \neq \ell, 1 \leq v \leq D-1$. From 1.9 , using the basic properties of determinants and $(2.2)$, it follows that

$$
\begin{aligned}
& \Delta_{r, D}(\underline{\alpha})= \\
& \left|\begin{array}{cccccccc}
\frac{\tilde{B}_{\alpha_{1}}\left(\frac{1}{D}\right)}{\alpha_{1}} & \ldots & \frac{\tilde{B}_{\alpha_{1}}\left(\frac{D-1}{D}\right)}{\alpha^{\prime}} & \frac{D^{\alpha_{1}} B_{\alpha_{1}}}{\alpha_{1}} & \ldots & \frac{\tilde{B}_{\alpha_{1}+r-1}\left(\frac{1}{D}\right)}{\alpha_{1}+r-1} & \ldots & \frac{D^{\alpha_{1}} B_{\alpha_{1}+r-1}}{\alpha_{1}+r-1} \\
\frac{\tilde{B}_{\alpha_{2}}\left(\frac{1}{D}\right)}{\alpha_{2}} & \ldots & \frac{\tilde{B}_{\alpha_{2}}\left(\frac{D-1}{D}\right)}{\alpha_{2}} & \frac{D^{\alpha_{2}} B_{\alpha_{2}}}{\alpha_{2}} & \ldots & \frac{\tilde{B}_{\alpha_{2}+r-1}\left(\frac{1}{D}\right)}{\alpha_{2}+r-1} & \ldots & \frac{D^{\alpha_{2}} B_{\alpha_{2}+r-1}}{\alpha_{2}+r-1} \\
\vdots & \ddots & \vdots & \vdots & \ddots & \vdots & \ddots & \vdots \\
\frac{\tilde{B}_{\alpha_{r D}}\left(\frac{1}{D}\right)}{\alpha_{r D}} & \ldots & \frac{\tilde{B}_{\alpha_{r D}}\left(\frac{D-1}{D}\right)}{\alpha_{r D}} & \frac{D^{\alpha_{r D}} B_{\alpha_{r D}}}{\alpha_{r D}} & \ldots & \frac{\tilde{B}_{\alpha_{r D}+r-1}\left(\frac{1}{D}\right)}{\alpha_{r D}+r-1} & \ldots & \frac{D^{\alpha_{r D} B_{\alpha_{r D}+r-1}}}{\alpha_{r D}+r-1}
\end{array}\right|
\end{aligned}
$$

Proposition 3.2. With the above assumptions, we have that $\Delta_{r, D}(\underline{\alpha}) \neq 0$ if and only if $\tilde{\Delta}_{r, D}(\underline{\alpha}) \neq 0$.

Proof. The conclusion follows from (3.2), 3.4), 3.5), (3.6), and (3.7), using an argument similar to that in the proof of Proposition 3.1 
Proposition 3.3 (Case $D=2$ ). With the above assumptions, $\Delta_{r, 2}(\underline{\alpha}) \neq 0$.

Proof. By Proposition 3.2 it is enough to prove that $\tilde{\Delta}_{r, 2}(\underline{\alpha}) \neq 0$. We have that

$$
\tilde{\Delta}_{r, 2}(\underline{\alpha})=\left|\begin{array}{cccc}
\frac{\tilde{B}_{\alpha_{1}}\left(\frac{1}{2}\right)}{\alpha_{1}} & \frac{\tilde{B}_{\alpha_{1}+1}\left(\frac{1}{2}\right)}{\alpha_{1}+1} & \ldots & \frac{\tilde{B}_{\alpha_{1}+r-1}\left(\frac{1}{2}\right)}{\alpha_{1}+r-1} \\
\frac{\tilde{B}_{\alpha_{2}\left(\frac{1}{2}\right)}}{\alpha_{2}} & \frac{\tilde{B}_{\alpha_{2}+1}\left(\frac{1}{2}\right)}{\alpha_{2}+1} & \ldots & \frac{\tilde{B}_{\alpha_{2}+r-1}\left(\frac{1}{2}\right)}{\alpha_{2}+r-1} \\
\vdots & \vdots & \ddots & \vdots \\
\frac{\tilde{B}_{\alpha_{r}\left(\frac{1}{2}\right)}}{\alpha_{r}} & \frac{\tilde{B}_{\alpha_{r}+1}\left(\frac{1}{2}\right)}{\alpha_{1}+1} & \ldots & \frac{\tilde{B}_{\alpha_{r}+r-1}\left(\frac{1}{2}\right)}{\alpha_{r}+r-1}
\end{array}\right|
$$

We choose $\alpha_{j}:=2^{j+t}-j+1$, where $2^{t} \geq r$. From 2.3 and Lemma 2.1 (1) it follows that $v_{2}\left(\tilde{B}_{\alpha_{j}+j-1}\left(\frac{1}{2}\right)\right)=0, \quad v_{2}\left(\tilde{B}_{\alpha_{j}+\ell-1}\left(\frac{1}{2}\right)\right) \geq 0, \quad$ for all $1 \leq j, \ell \leq r, j \neq \ell$.

On the other hand,

$$
j+t=v_{2}\left(\alpha_{j}+j-1\right)>v_{2}\left(\alpha_{j}+\ell-1\right), \quad \text { for all } 1 \leq j, \ell \leq r, j \neq \ell .
$$

From 3.8, 3.9, and 3.10 it follows that

$$
v_{2}\left(\tilde{\Delta}_{r, 2}(\underline{\alpha})\right)=v_{2}\left(\prod_{j=1}^{r} \frac{\tilde{B}_{\alpha_{j}+j-1}\left(\frac{1}{2}\right)}{\alpha_{j}+j-1}\right)=-r t-\left(\begin{array}{l}
r \\
2
\end{array}\right) .
$$

Hence, $\tilde{\Delta}_{r, 2}(\underline{\alpha}) \neq 0$, as required.

In the following, we assume that $D \geq 3$. Let $N:=\left\lfloor\frac{(D-1) r}{2}\right\rfloor$. We also assume that $\alpha_{t}$ is odd for all $1 \leq t \leq N$, and $\alpha_{t}$ is even for all $N+1 \leq t \leq r(D-1)$. Let $k:=\left\lfloor\frac{D-1}{2}\right\rfloor$ and $\bar{k}=\left\lceil\frac{D-1}{2}\right\rceil$. From 2.1 and 2.2 it follows that

$$
\tilde{B}_{\alpha_{t}+j-1}\left(\frac{D-v}{D}\right)+\tilde{B}_{\alpha_{t}+j-1}\left(\frac{v}{D}\right)= \begin{cases}0, & \text { if } \alpha_{t}+j-1 \text { is odd } \\ 2 \tilde{B}_{\alpha_{t}+j-1}\left(\frac{v}{D}\right), & \text { if } \alpha_{t}+j-1 \text { is even }\end{cases}
$$

for all $1 \leq t \leq r(D-1), 1 \leq v \leq \bar{k}$, and $1 \leq j \leq r$. We consider the determinants

$$
\tilde{\Delta}_{r, D}^{\prime}(\underline{\alpha}):=\left|\begin{array}{ccccccc}
\frac{\tilde{B}_{\alpha_{1}}\left(\frac{1}{D}\right)}{\alpha_{1}} & \ldots & \frac{\tilde{B}_{\alpha_{1}}\left(\frac{k}{D}\right)}{\alpha_{1}} & \frac{\tilde{B}_{\alpha_{1}+1}\left(\frac{1}{D}\right)}{\alpha_{1}+1} & \ldots & \frac{\tilde{B}_{\alpha_{1}+1}\left(\frac{\bar{k}}{D}\right)}{\alpha_{1}+1} & \ldots \\
\frac{\tilde{B}_{\alpha_{2}}\left(\frac{1}{D}\right)}{\alpha_{2}} & \ldots & \frac{\tilde{B}_{\alpha_{2}}\left(\frac{k}{D}\right)}{\alpha_{2}} & \frac{\tilde{B}_{\alpha_{2}+1}\left(\frac{1}{D}\right)}{\alpha_{2}+1} & \ldots & \frac{\tilde{B}_{\alpha_{2}+1}\left(\frac{\bar{k}}{D}\right)}{\alpha_{2}+1} & \ldots \\
\vdots & \ddots & \vdots & \vdots & \ddots & \vdots & \ddots \\
\frac{\tilde{B}_{\alpha_{N}\left(\frac{1}{D}\right)}}{\alpha_{N}} & \ldots & \frac{\tilde{B}_{\alpha_{N}}\left(\frac{k}{D}\right)}{\alpha_{N}} & \frac{\tilde{B}_{\alpha_{N+1}\left(\frac{1}{D}\right)}}{\alpha_{N+1}} & \ldots & \frac{\tilde{B}_{\alpha_{N+1}\left(\frac{\bar{k}}{D}\right)}}{\alpha_{N+1}} & \ldots
\end{array}\right|
$$


and

$$
\tilde{\Delta}_{r, D}^{\prime \prime}(\underline{\alpha}):=\left|\begin{array}{ccccccc}
\frac{\tilde{B}_{\alpha_{N+1}\left(\frac{1}{D}\right)}}{\alpha_{N+1}} & \ldots & \frac{\tilde{B}_{\alpha_{N+1}\left(\frac{\bar{k}}{D}\right)}}{\alpha_{N+1}} & \frac{\tilde{B}_{\alpha_{N+1}+1}\left(\frac{1}{D}\right)}{\alpha_{N+1}+1} & \ldots & \frac{\tilde{B}_{\alpha_{N+1}+1}\left(\frac{k}{D}\right)}{\alpha_{N+1}+1} & \ldots \\
\frac{\tilde{B}_{\alpha_{N+2}\left(\frac{1}{D}\right)}}{\alpha_{N+2}} & \ldots & \frac{\tilde{B}_{\alpha_{N+2}\left(\frac{\bar{k}}{D}\right)}}{\alpha_{N+2}} & \frac{\tilde{B}_{\alpha_{N+2}+1}\left(\frac{1}{D}\right)}{\alpha_{N+2}+1} & \ldots & \frac{\tilde{B}_{\alpha_{N+2}+1}\left(\frac{k}{D}\right)}{\alpha_{N+2}+1} & \ldots \\
\vdots & \ddots & \vdots & \vdots & \ddots & \vdots & \ddots \\
\frac{\tilde{B}_{\alpha_{r D-r}\left(\frac{1}{D}\right)}}{\alpha_{r D-r}} & \ldots & \frac{\tilde{B}_{\alpha_{r D-r}\left(\frac{\bar{k}}{D}\right)}}{\alpha_{r D-r}} & \frac{\tilde{B}_{\alpha_{r D-r}+1}\left(\frac{1}{D}\right)}{\alpha_{r D-r}+1} & \ldots & \frac{\tilde{B}_{\alpha_{r D-r}+1}\left(\frac{k}{D}\right)}{\alpha_{r D-r}+1} & \ldots
\end{array}\right| .
$$

Proposition 3.4. With the above assumptions, we have that

$$
\tilde{\Delta}_{r, D}(\underline{\alpha})=C \tilde{\Delta}_{r, D}^{\prime}(\underline{\alpha}) \tilde{\Delta}_{r, D}^{\prime \prime}(\underline{\alpha}),
$$

where $C \neq 0$. In particular, if $\tilde{\Delta}_{r, D}^{\prime}(\underline{\alpha}) \neq 0$ and ${\tilde{\Delta^{\prime \prime}}}_{r, D}(\underline{\alpha}) \neq 0$ then $\tilde{\Delta}_{r, D}(\underline{\alpha}) \neq 0$.

Proof. In $(3.2)$, we add the $(j+t r)$-th column over the $(D-j+t r)$-th column, where $1 \leq j \leq k$ and $0 \leq t \leq r-1$. The conclusion follows from (3.11), (3.12), and (3.13) using the basic properties of determinants.

\section{Proof of Theorem 1.2}

The case $D=1$ was proved in Proposition 3.1 Also, the case $D=2$ was proved in Proposition 3.3. Assume that $D:=p>2$ is a prime number. Let $k:=\left\lfloor\frac{p-1}{2}\right\rfloor$. According to Proposition 3.4 it is enough to prove that $\tilde{\Delta}_{r, p}^{\prime}(\underline{\alpha}) \neq 0$ and ${\tilde{\Delta^{\prime \prime}}}_{r, p}(\underline{\alpha}) \neq 0$. Let

$$
t_{1}<t_{2}<\cdots<t_{r}
$$

be a sequence of positive integers, such that $t_{1}>\log _{p}(r-1):=$ the logarithm of $r-1$ to base $p$. We define

$$
\alpha_{j+(s-1) k}:= \begin{cases}2 j p^{t_{s}}-s+1, & \text { if } s \text { is even; } \\ (2 j-1) p^{t_{s}}-s+1, & \text { if } s \text { is odd }\end{cases}
$$

for all $1 \leq s \leq r, 1 \leq j \leq k$. From (4.1) and 4.2 it follows that

$$
\begin{aligned}
v_{p}\left(\alpha_{j+(s-1) k}+s-1\right)=t_{s}, & \text { for all } 1 \leq s \leq r, 1 \leq j \leq k \\
v_{p}\left(\alpha_{j+(s-1) k}+\ell\right)<t_{1}, & \text { for all } 1 \leq s \leq r, 1 \leq j \leq k \\
& \text { and } 0 \leq \ell \leq r-1 \text { with } \ell \neq s-1 .
\end{aligned}
$$

On the other hand, from Lemma 2.1(2) it follows that

$$
B_{\alpha_{j}}\left(\frac{v}{p}\right) \equiv v^{\alpha_{j}} \quad(\bmod p), \quad \text { for all } 1 \leq j \leq r p .
$$

From 4.3, 4.4 , and 4.5 it follows that 


$$
\begin{array}{r}
v_{p}\left(\frac{\widetilde{B}_{\alpha_{j+(s-1) k}+s-1}\left(\frac{v}{p}\right)}{\alpha_{j+(s-1) k}+s-1}\right)=-t_{s}, \quad \text { for all } 1 \leq s \leq r, 1 \leq j, v \leq k, \\
v_{p}\left(\frac{\widetilde{B}_{\alpha_{j+(s-1) k}+\ell\left(\frac{v}{p}\right)}}{\alpha_{j+(s-1) k}+\ell}\right)>-t_{1}, \quad \text { for all } 1 \leq s \leq r, 1 \leq j, v \leq k, \\
\quad 0 \leq \ell \leq r-1 \text { with } \ell \neq s-1 .
\end{array}
$$

We consider the determinants

$$
M_{s}:=\operatorname{det}\left(\widetilde{B}_{\alpha_{j+(s-1) k}+s-1}\left(\frac{v}{p}\right)\right)_{1 \leq j, v \leq k}, \quad 1 \leq s \leq r .
$$

From (4.5) it follows that

$$
\begin{aligned}
& M_{s} \equiv \operatorname{det}\left(v^{2 j p^{t_{s}}}\right)_{1 \leq j, v \leq k} \equiv \operatorname{det}\left(v^{2 j}\right)_{1 \leq j, v \leq k} \quad(\bmod p) \quad \text { for } s \text { even, } \\
& M_{s} \equiv \operatorname{det}\left(v^{2(j-1) p^{t_{s}}}\right)_{1 \leq j, v \leq k} \equiv \operatorname{det}\left(v^{2 j-1}\right)_{1 \leq j, v \leq k} \quad(\bmod p) \quad \text { for } s \text { odd } .
\end{aligned}
$$

On the other hand, using the Vandermonde formula, we have

$$
\begin{aligned}
\operatorname{det}\left(v^{2 j}\right)_{1 \leq j, v \leq k} & =v^{2} \prod_{1 \leq i<j \leq k}(j-i)(j+i) \not \equiv 0 \quad(\bmod p), \\
\operatorname{det}\left(v^{2 j-1}\right)_{1 \leq j, v \leq k} & =v \prod_{1 \leq i<j \leq k}(j-i)(j+i) \not \equiv 0 \quad(\bmod p) .
\end{aligned}
$$

From 4.9, 4.10, 4.11, and 4.12 it follows that

$$
v_{p}\left(M_{s}\right)=0, \quad \text { for all } 1 \leq s \leq r .
$$

Hence, it follows that $M_{s} \neq 0$. From (3.12), 4.6), 4.7), 4.8), and 4.13) it follows that

$$
v_{p}\left(\tilde{\Delta}_{r, p}^{\prime}(\underline{\alpha})\right)=-\left(t_{1}+t_{2}+\cdots+t_{r}\right) k
$$

Therefore, ${\tilde{\Delta^{\prime}}}_{r, p}^{\prime}(\underline{\alpha}) \neq 0$. Similarly, one can prove that ${\tilde{\Delta^{\prime \prime}}}_{r, p}(\underline{\alpha}) \neq 0$.

\section{ACKNOWLEDGMENT}

I would like to express my gratitude to Florin Nicolae for the valuable discussions regarding this paper.

\section{REFERENCES}

[1] G. Almkvist and A. Meurman, Values of Bernoulli polynomials and Hurwitz's zeta function at rational points, C. R. Math. Rep. Acad. Sci. Canada 13 (1991), no. 2-3, 104-108. MR 1112244

[2] T. M. Apostol, Introduction to analytic number theory, Undergraduate Texts in Mathematics, Springer-Verlag, New York, 1976. MR 0434929

[3] E. W. Barnes, On the theory of the multiple gamma function, Trans. Camb. Philos. Soc. 19 (1904), 374-425.

[4] A. Bayad and M. Beck, Relations for Bernoulli-Barnes numbers and Barnes zeta functions, Int. J. Number Theory 10 (2014), no. 5, 1321-1335. MR 3231418 
[5] E. T. Bell, Interpolated denumerants and Lambert series, Amer. J. Math. 65 (1943), 382-386. MR 0009043

[6] M. Cimpoeaş and F. Nicolae, On the restricted partition function, Ramanujan J. 47 (2018), no. 3, 565-588. MR 3874808 .

[7] M. Cimpoeaş and F. Nicolae, Corrigendum to "On the restricted partition function", $R a-$ manujan J. 49 (2019), no. 3, 699-700. MR 3979698

[8] M. Cimpoeaş, On the restricted partition function via determinants with Bernoulli polynomials, Mediterr. J. Math. 17 (2020), no. 2, Art. 51, 19 pp. MR 4067184

[9] T. Clausen, Lehrsatz aus einer Abhandlung über die Bernoullischen Zahlen, Astr. Nachr. 17 (1840), 351-352.

[10] F. R. Olson, Some determinants involving Bernoulli and Euler numbers of higher order, Pacific J. Math. 5 (1955), 259-268. MR 0069125.

[11] T. Popoviciu, Asupra unei probleme de partiţie a numerelor, Acad. R. P. Române. Fil. Cluj. Stud. Cerc. Şti. 4 (1953), no. 1-2, 7-58.

[12] S. N. M. Ruijsenaars, On Barnes' multiple zeta and gamma functions, Adv. Math. 156 (2000), no. 1, 107-132. MR 1800255.

[13] M. Spreafico, On the Barnes double zeta and Gamma functions, J. Number Theory 129 (2009), no. 9, 2035-2063. MR 2528052

[14] K. G. C. Staudt, Beweis eines Lehrsatzes, die Bernoullischen Zahlen betreffen, J. Reine Angew. Math. 21 (1840), 372-374. MR 1578267

[15] J. J. Sylvester, On the partition of numbers, Quart. J. Pure Appl. Math. 1 (1857), 141-152. https://hdl.handle.net/2027/uc1.\$b417523?urlappend=\%3Bseq=161

\section{Mircea Cimpoeaş}

Simion Stoilow Institute of Mathematics, Research unit 5, P.O. Box 1-764, 014700 Bucharest, Romania

Politehnica University of Bucharest, Faculty of Applied Sciences, Department of Mathematical Methods and Models, 060042 Bucharest, Romania

mircea.cimpoeas@imar.ro

Received: April 27, 2019

Accepted: October 25, 2019 